\title{
Characteristics of Patients with Hearing Aids according to the Degree and Pattern of Hearing Loss
}

\author{
Young Seok Byun ${ }^{1}$, Sung Su Kim², Sang Hyun Park ${ }^{1}$, Eun Bin Park, \\ Ho Joong Kim${ }^{1}$, Sang Hoon Kim¹, and Seung Geun Yeo ${ }^{1}$ \\ ${ }^{1}$ Department of Otorhinolaryngology-Head \& Neck Surgery, School of Medicine, Kyung Hee University, Seoul, \\ ${ }^{2}$ Department of Biochemistry and Molecular Biology, Medical Science and Engineering Research Center for Bioreaction \\ to Reactive Oxygen Species, BK-21, School of Medicine, Kyung Hee University, Seoul, Korea
}

\begin{abstract}
Received March 10,2016
Revised May 31, 2016

Accepted June 7, 2016
\end{abstract}

Background and Objectives: This study was designed to assess the characteristics of patients according to the degree and audiogram shape of hearing loss and the association of these characteristics with hearing aids $(\mathrm{HA})$ choice, return rate, and cause of return. Subjects and Methods: This study included 460 individuals who received HAs from 2011 to 2015. The relationships between type of HA and age, primary and accompanying symptoms, HA choice and return and cause of return were evaluated according to the degree and pattern of hearing loss. Results: HA type did not differ significantly according to the degree and pattern of hearing loss. Intensity of hearing loss was greater in male than in female $(p<0.05)$. Open and completely-in-canal types of HA decreased with age $(p<0.05)$. As degree of hearing loss intensified, behind-the-ear and in-the-ear types increased and Open type decreased $(p<0.05)$. The HA return rate was $9.7 \%$, but was not associated with degree or pattern of hearing loss. The main causes of HA return were costs, psychological fears and adaptive failure. Conclusions: Choice of $\mathrm{HA}$ is affected by age, sex, and degree and pattern of hearing loss. HA for hearing rehabilitation in patients with hearing loss can be personalized according to each patient's characteristics and tendencies.

J Audiol Otol 2016;20(3):146-152

KEY WORDS: Hearing aids · Degree of hearing loss · Pattern of hearing loss Type of hearing loss.

\section{Introduction}

Hearing loss is caused by a combination of factors, including genetic characteristics, noise exposure, and aging. In addition to causing communication disorders, hearing loss has been associated with various conditions, including depression, dementia, and tinnitus, as well as reducing quality of life $[1,2]$. Increases in life expectancy result in higher percentages of individuals with hearing loss. Hearing loss in elderly individuals may cause behavioral and psychosocial disorders and reduce confidence, as well as being likely to trigger psychological alienation, depression and anxiety. Hearing loss in

This is an Open Access article distributed under the terms of the Creative Commons Attribution Non-Commercial License (http://creativecommons.org/licenses/by-nc/3.0/) which permits unrestricted non-commercial use, distribution, and reproduction in any medium, provided the original work is properly cited. children may have deleterious effects on academic ability and linguistic development [3-5].

Clinician awareness of the symptoms of hearing loss at an early stage can lead to hearing assessment, diagnosis and evaluation, and hearing rehabilitation [1,2]. Hearing aids can overcome hearing problems, resulting in greater adaptation to everyday life [5]. Even if the degree of hearing loss is not so severe and a patient does not believe that wearing a hearing aid would result in significant improvement, the effects of wearing a hearing aid should be tested [6].

To date, few studies have assessed the satisfaction level or the association of patient characteristics and audiogram shape and degree of hearing loss with the selection of a hearing aid. This study therefore assessed the sex, age, and primary and secondary symptoms, as well as the audiogram shape of hearing aid selected, return rate of hearing aids, and reasons for 
return, in patients prescribed hearing aids at our institution between 2011 and 2015 relative to the degree and audiogram shape of hearing loss.

\section{Subjects and Methods}

\section{Subjects and study design}

The study population consisted of 472 patients who visited the Department of Otorhinolaryngology from January 2011 to June 2015 and 12 patients who were excluded for several reasons, including loss to follow up and death. Thus, $460 \mathrm{pa}-$ tients who were prescribed hearing aids were included in the study (Fig. 1). All patients were assessed by pure-tone audiometry, with the degree of hearing loss in each classified as mild, moderate-severe or severe. Patients were also classified as having a flat, descending, ascending, concave or convex pattern of hearing loss; and, depending on their profile of air and bone conduction, as having conductive, sensorineural, or mixed hearing loss. Age, sex, primary and secondary symptoms, and audiogram shape of hearing aid prescribed were recorded, as were the reason for return of the hearing aid, if applicable.

\section{Objective measurements}

\section{Pure-tone audiometry}

Pure-tone audiometry (PTA) was performed to determine the pattern and type of hearing loss and the results of air and bone conduction tests. Each threshold and each mean threshold value calculated by a 6 part-partition method were recorded at 125, 256, 500, 1, 2, 3, 4, and $8 \mathrm{kHz}$. The mean threshold value from the 6 part-partition method was calculated as [500 $\mathrm{Hz}+(2 \times 1,000 \mathrm{~Hz})+(2 \times 2,000 \mathrm{~Hz})+4,000 \mathrm{~Hz}] / 6$. The degree of hearing loss was classified as mild ( $25 \mathrm{~dB}$ to $40 \mathrm{~dB})$, moderate-to-severe ( $41 \mathrm{~dB}$ to $70 \mathrm{~dB})$, or severe $(71 \mathrm{~dB}$ to 90 dB) [7]. Patterns of hearing loss, whether flat, ascending, descending, concave or convex, were determined as described previously $[8,9]$.

If a comparison of air and bone conduction curves showed a reduction in the former but normal bone conduction on PTA, the loss was defined as conductive type. If both the air and bone hearing thresholds had deteriorated to the same extent, with the air hearing threshold being greater than $10 \mathrm{~dB}$, the loss was defined as mixed type [7].

\section{Hearing aids}

Patients who were prescribed hearing aids were evaluated based on three subjective PTA tests and speech audiometry, most comfortable loudness, uncomfortable loudness, and dynamic range enforcement. Taking into account the age and occupation of the patients, we explained to them the suitable types of hearing aid, their shapes, prices and disadvantages.

After prescription of the hearing aid, a fitting and verification process was followed to fit the outer ear canal if molding was required. After one month, the hearing aids were modified based on patient feedback. After three months, PTA was performed and we checked whether the hearing aid had been beneficial used or not. If a patient wanted to return the device, we investigated the underlying reasons.

\section{Statistical analysis}

All statistical analyses were performed using SPSS 18.0 software (SPSS Inc., Chicago, IL, USA). The mean of the three groups was compared by one-way ANOVA, while frequencies were compared pairwise by chi-square tests. A $p$ value less than 0.05 was defined as statistically significant.

\section{Results}

The 460 patients consisted of 203 male (44.1\%) and 257 female $(55.9 \%)$. When divided by age, the number of patients increased with age. But the difference was not significant $(p>0.05)$. Although the intensity of hearing loss was unrelated to age, it was associated with an increased proportion of male patients and decreased proportion of female patients $(p<0.05)$. In contrast, groups classified by audiogram shape of hearing loss were not associated with age or sex (Table 1) $(p>0.05)$. Hearing loss and tinnitus were the main symptoms in patients with mild, moderate-severe, and severe hearing

Table 1. Age, degree and audiogram pattern of hearing loss by gender in patients wearing hearing aids

\begin{tabular}{lcccc}
\hline & $\begin{array}{c}\text { Male } \\
(\mathrm{n}=203)\end{array}$ & $\begin{array}{c}\text { Female } \\
(\mathrm{n}=257)\end{array}$ & $\begin{array}{c}\text { Total } \\
(\mathrm{n}=460)\end{array}$ & p-value \\
\hline $\begin{array}{l}\text { Age, yr (\%) } \\
<18\end{array}$ & $3(0.6)$ & $2(0.5)$ & $5(1.1)$ & 0.477 \\
$19-40$ & $12(2.6)$ & $15(4.4)$ & $27(7)$ & \\
$41-69$ & $89(19.3)$ & $100(20.8)$ & $189(41.1)$ & \\
$>70$ & $99(21.5)$ & $140(30.5)$ & $239(52)$ & \\
Degree of hearing loss (\%) & & & $0.009^{*}$ \\
Mild & $6(1.3)$ & $25(5.4)$ & $31(6.7)$ & \\
Moderate-severe & $159(34.5)$ & $196(42.7)$ & $355(77.2)$ & \\
Severe & $38(8.1)$ & $36(8)$ & $74(16.1)$ & \\
Audiogram pattern & & & & 0.134 \\
of hearing loss (\%) & & & & \\
Flat & $31(6.7)$ & $43(9.4)$ & $74(16.1)$ & \\
Descending & $146(31.7)$ & $174(37.9)$ & $320(69.6)$ & \\
Ascending & $7(1.5)$ & $4(0.9)$ & $11(2.4)$ & \\
Concave & $3(0.6)$ & $14(3.1)$ & $17(3.7)$ & \\
Convex & $16(3.4)$ & $22(4.9)$ & $36(8.3)$ & \\
\hline
\end{tabular}

* $p<0.05 . p$-value is gender ratio's chi-squared test 
oss; as the degree of hearing loss became more intense, however, hearing loss tended to become the main symptom, while tinnitus tended to decrease. Hearing loss and tinnitus were also the main symptoms in all five groups of patients divided by audiogram shape. Associated symptoms were also assessed in each group of patients classified by degree of hearing loss and audiogram shape. Tinnitus, aural fullness, hearing loss and dizziness were the most frequent associated symptoms in all three groups classified by intensity of hearing loss and all five groups classified by audiogram shape. Intensity of hearing loss was associated with an increased selection of the behind-the-ear (BTE) and in-the-ear (ITE) types of hearing aids $(p<0.05)$ and a reduced selection of Open type hearing aids $(p<0.05)$ (Fig. 2$)$. The selection of Open type hearing aids differed in patients with ascending and flat hearing loss and in patients with ascending and convex hearing loss $(p<0.05)$ (Fig. 3). The Open type was more frequent in patients $<18$ than $>19$ to $<40$ years old, but the difference was not significant. Increased age was associated with significantly less frequent use of Open and completely-in-cancal (CIC) types of hearing aids $(p<0.05)$ (Fig. 4).

The overall hearing aid return rate was $9.7 \%$. This rate was not affected by the intensity of hearing loss (Fig. 5). The return rate was significantly lower in patients with flat than with descending and convex shaped audiograms, and was significantly lower in patients with descending than Convex

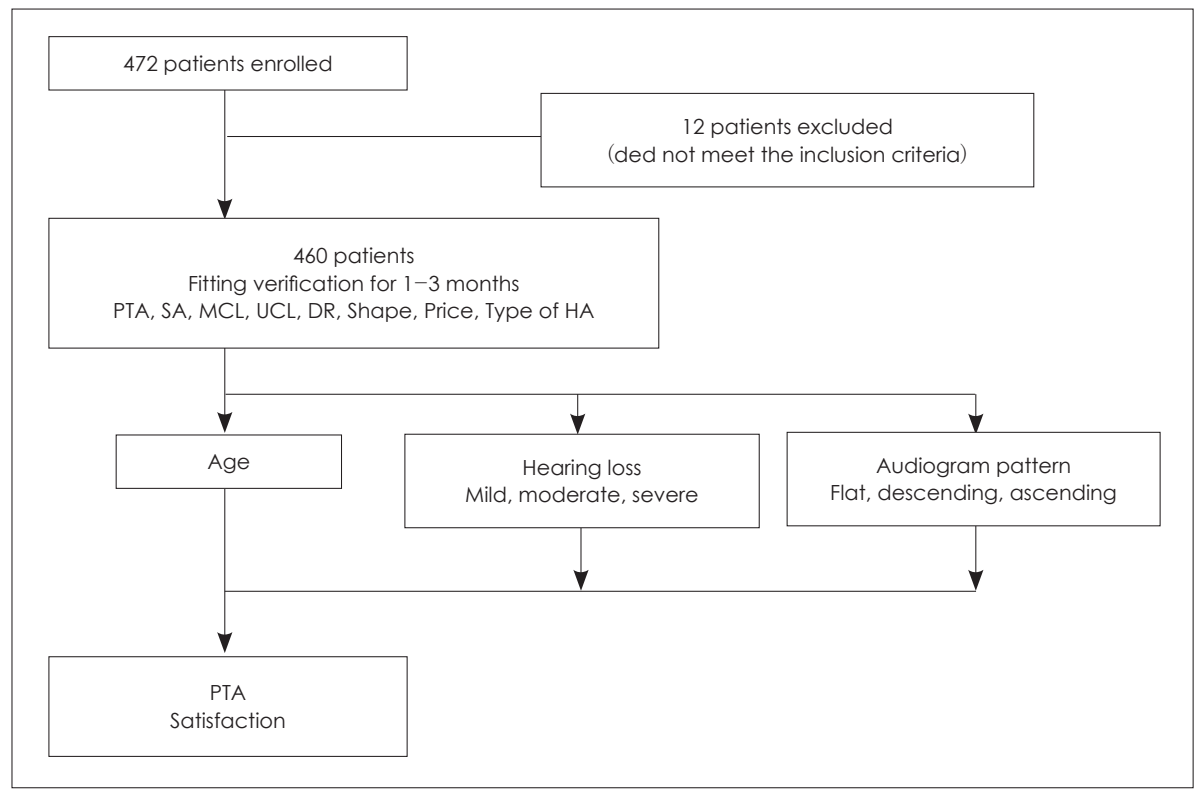

Fig. 1. Overview of patient enrollment. PTA: pure-tone audiometry, SA: speech audiometry, MCL: most comfortable loudness, UCL: uncomfortable loudness, DR: dynamic range, $\mathrm{HA}$ : hearing aids.

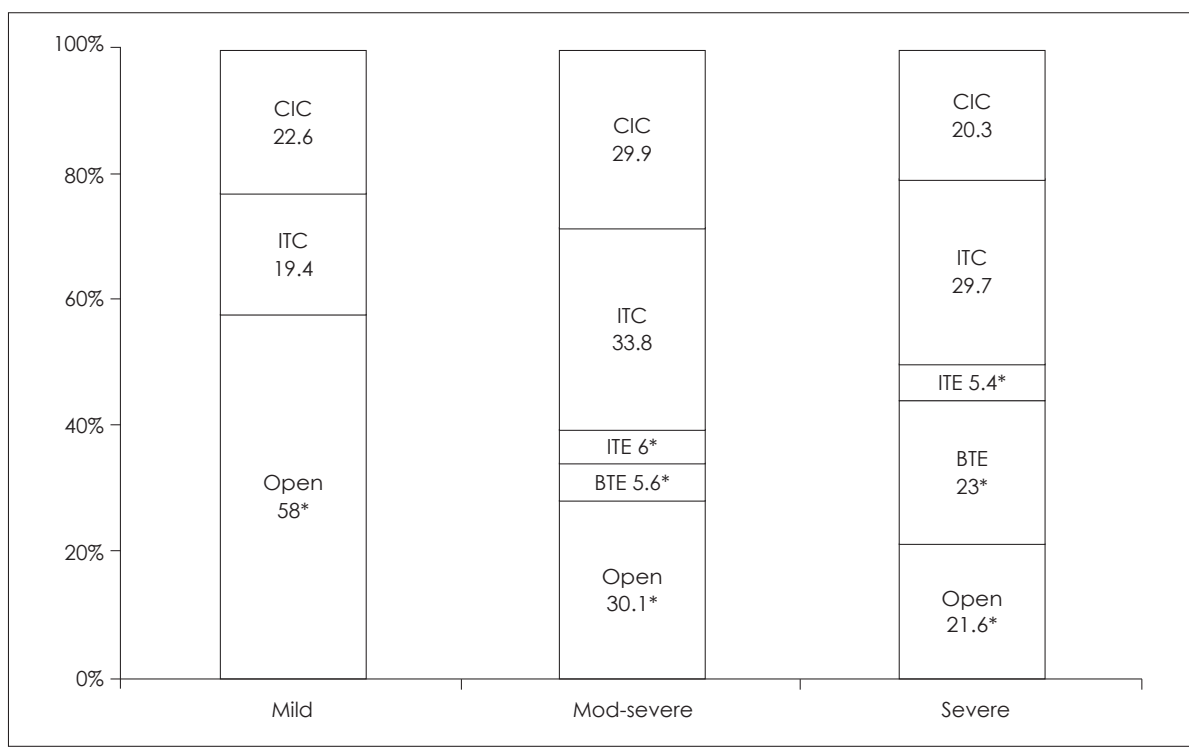

Fig. 2. Choice of hearing aids in patients classified by degree of hearing loss. ${ }^{*} p<0.05$ (linear by linear association). CIC: completely-in-canal, ITC: in-the-ear, BTE: behind-the-ear. 
Fig. 3. Choice of hearing aids in patients classified by audiogram pattern of hearing loss (chi-squared test). CIC: completely-in-canal, ITC: in-the-ear, BTE: behind-the-ear.
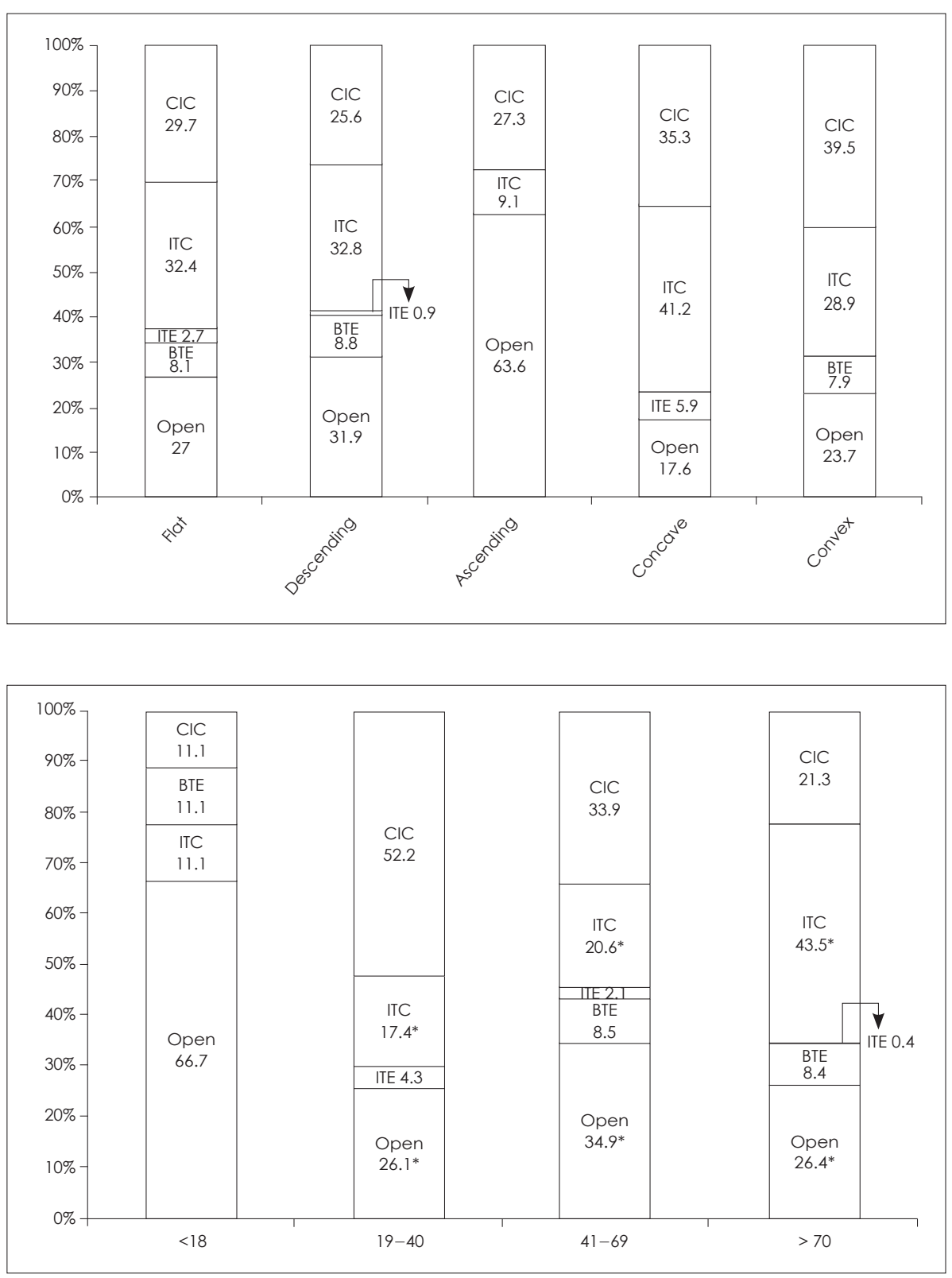

Fig. 4. Choice of hearing aids in patients classified by age of hearing loss. ${ }^{*} p<0.05$ (chi-squared test). CIC: completely-in-canal, ITC: in-the-ear, BTE: behind-the-ear. patients without tinnitus, only 9 patients $(6.4 \%)$ returned their hearing aids. These findings indicate that the rate of hearing aid return by symptomatic patients was not significantly related to whether the patient experienced tinnitus $(p=0.935)$ (Table 3).

\section{Discussion}

This study examined the characteristics of patients with hearing aids according to the degree and pattern of hearing loss. The investigation included both quantitative and qualitative measures and experience in a daily life setting. In particular, we examined the relation between the aspects of daily life 


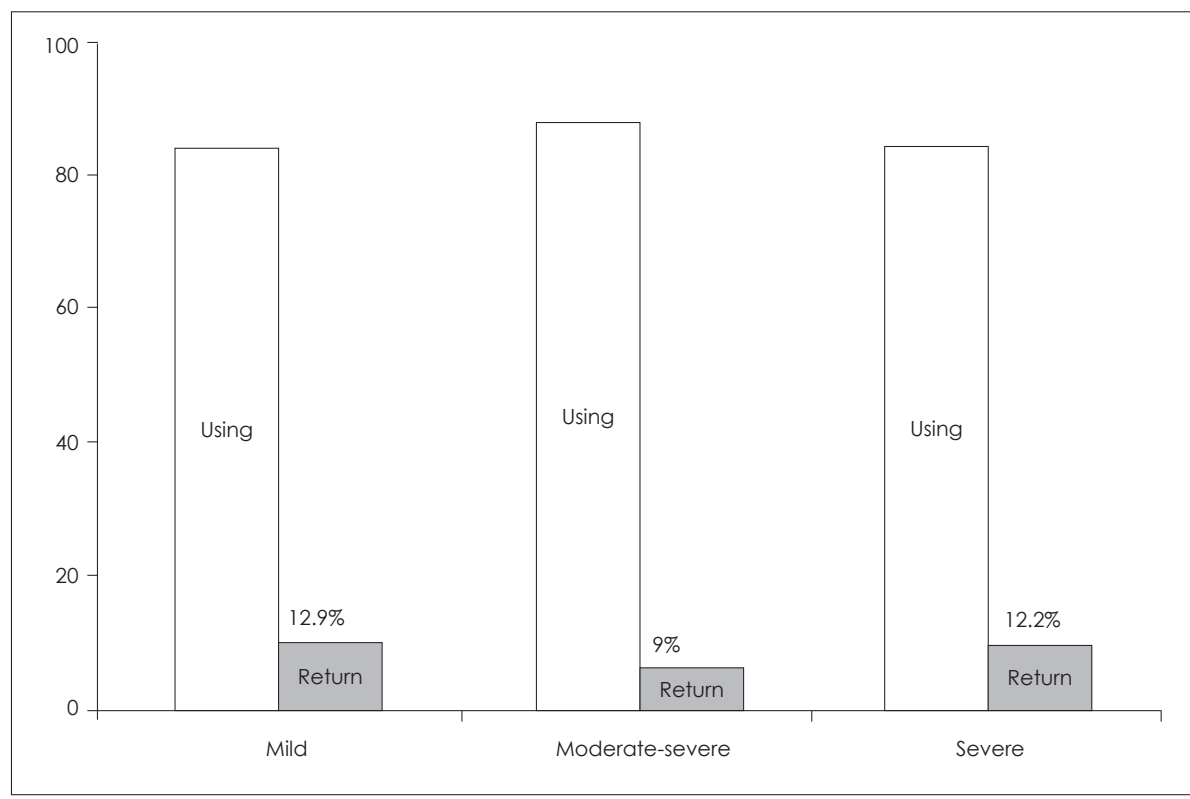

Fig. 5. Hearing aid return rate in patients classified by degree of hearing loss.

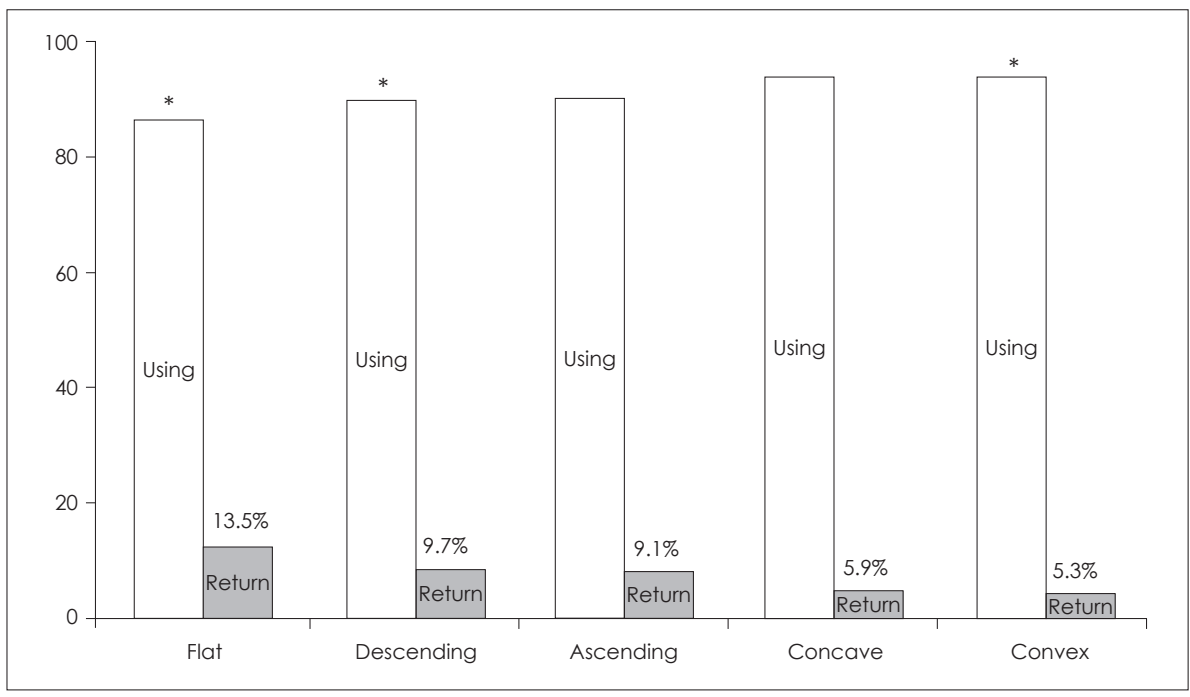

Fig. 6. Hearing aid return rate in patients classified by audiogram shape of hearing loss. ${ }^{*} p<0.05$ (chi-squared test).

Table 2. Reasons for return of hearing aids

\begin{tabular}{lc}
\hline Unknown (patient cancellation) & $23 / 51(45 \%)$ \\
Financial reasons & $7 / 51(13.3 \%)$ \\
Ineffectiveness & $7 / 51(13.3 \%)$ \\
Adaptation failure & $7 / 51(13.3 \%)$ \\
Feedback/noise & $4 / 51(7.1 \%)$ \\
Headache/dizziness & $2 / 51(3 \%)$ \\
Frequently out of order & $1 / 51(2 \%)$ \\
\hline
\end{tabular}

and hearing aid return rate. The included patients had not been previously exposed to specific hearing aids, and we did not attempt to influence the patient decision regarding the type of hearing aid. Rather, patients were counseled about the choice of hearing aids and given advice on how to use the de-
Table 3. Proportion of hearing aid-related symptom and hearing aid return

\begin{tabular}{lccc}
\hline & Hearing aid use & Hearing aid return & $p$-value \\
\hline Symptom (+) & $242(92.7 \%)$ & $19(7.3 \%)$ & $0.039 *$ \\
Symptom (-) & $173(86.9 \%)$ & $26(13.1 \%)$ & \\
Tinnitus (+) & $131(93.6 \%)$ & $9(6.4 \%)$ & 0.935 \\
Tinnitus (-) & $140(93.3 \%)$ & $10(6.7 \%)$ & \\
\hline * $p<0.05 . p$-value by the chi-squared tes ${ }^{\circ}$ &
\end{tabular}

vices to achieve optimal benefit.

The population of elderly individuals is increasing, prolonging average life expectancy and increasing the numbers of patients with hearing loss. In Korea, the proportion of the population aged over 65 years is increasing, such that it is 
predicted to be $14.5 \%$ by 2018 [10]. Concomitant with this increase in the number of elderly individuals, hearing aid technology has progressed in leaps and bounds, and hearing aid developers are continually striving to eliminate patient discomfort. However, $3 \%$ to $16 \%$ of patients prescribed hearing aids return the devices in countries such as the USA and other European countries [11].

Hearing aids are important and effective rehabilitation methods for patients with mild and moderate-severe hearing loss, in most patients with sensorineural hearing loss and in some with conductive hearing loss $[5,12]$. Hearing aids are recommended proactively during initial stages of hearing loss [13]. A survey in the United States in 2001 reported that $30 \%$ of individuals in their $70 \mathrm{~s}$ and $50 \%$ of those in their 80 s complained of hearing loss. Although the number of the patients with hearing aids also increased with age, the increased rate of hearing loss was not proportional to greater age $[1,14,15]$. Although several studies have reported that the prevalence of hearing loss in the general population in Korea has increased as the population ages, and that the prevalence in $20-30$ year old Korean males has increased due to military service, the present study found that the prevalence of hearing loss was similar in male and female in patients wearing hearing aids. The flat pattern of hearing loss was observed primarily in female while the descending pattern was present primarily in male. However, a survey suggested a high proportion of female had the descending pattern. This finding indicated that the high frequency steeply sloping type appeared primarily in men in both subtypes of the descending group whereas the high frequency gently sloping type appeared primarily in female [9]. The ascending pattern was rare in this study, being present in only $2.4 \%$ of patients, making it difficult to determine its significance [16-18].

In groups of patients classified by audiogram shape, the major symptoms were generally hearing loss and tinnitus. In patients with an ascending pattern, however, the major symptoms were hearing loss and aural fullness, with tinnitus considered an associated symptom in this group. Tinnitus is a major symptom in patients with severe damage to high frequency hearing ability. High frequency hearing ability is better preserved in patients with an ascending than other patterns of hearing loss $[19,20]$. In groups of patients classified by degree of hearing loss, the rate of tinnitus tends to increase as the degree of hearing loss intensifies, whereas the rates of aural fullness and otalgia tend to decrease as the degree of hearing loss intensifies; thus, progressive damage to hearing organs likely affects the acoustic and sensory nerves together [21]. Use of the BTE and ITC types of hearing aids tended to increase with age, This was thought to be due to the easier manipula- tion of these than other types of hearing aids for in patients who were too old were or had difficulty operating the hearing aids. More widespread use of the ITC than of the ITE type was considered to be due to aesthetic reasons [3,4,22]. A lesser degree of hearing loss was significantly associated with greater use of open type hearing aids, with more severe hearing loss associated with increased selection of the BTE and ITE types. Patients with mild hearing loss were prescribed all forms of hearing aids, especially the CIC and Open (receiver in the canal) types. Patients with moderate-severe and severe hearing loss tended to be older and tended to be prescribed the BTE and ITE $[2,22,23]$. When the choice of hearing aid was compared among the five groups classified by audiogram shape, we found that the open type was significantly more frequent in patients with ascending than flat type patterns, but was significantly less frequent in patients with convex than ascending patterns. Hearing ability in patients with ascending patterns of hearing loss showed a greater deterioration at low than at high frequency. These patients were more likely to be prescribed CIC than open types of hearing aids, because the former did not block the ears, whereas the latter showed poorer low-frequency amplification ability. Although many patients with the ascending pattern were prescribed CIC types of hearing aids, the prescription of open types in a large proportion of these patients may have been due to the limited number of patients. In the descending group, ITC type hearing aids had low band occlusion effects, resulting in an echo; although open type hearing aids were prescribed more widely than any other types, this study reported inconsistent results [22]. This was likely due to the visibility of open hearing aids outside the ear.

When we compared the choice of hearing aid among the five groups classified by audiogram shape, we found that the open type was significantly more frequent in patients with an ascending type than a flat type pattern and significantly less frequent in patients with a convex than an ascending pattern. However, due to the limited number of patients in the present work, further research on this topic is needed.

The hearing aids returned by the patients included not only those being used but those prescribed but cancelled prior to production. The overall return rate was $9.7 \%$, with no significant inter-group differences among patients classified by the intensity of hearing loss and by audiogram shape. The reasons for return of hearing aids were diverse, with half giving no reasons, as most were cancellations within 3 to 4 days of first request. This was unrelated to the degree of hearing loss and hearing loss itself, as well as to the degree of impairment felt by the patient, physical problems, psychological conditions, social status, or recommendations by others or family 
members. Economic circumstances were a significant cause of hearing aid return. Other reasons included difficulties in wearing, sense of burden, ineffectiveness, inconvenience, and worries about looking old or disabled. Patients also mistakenly thought that hearing aids would not help improve hearing ability or that the hearing aids would substantially reduce the quality of sound. The proper diagnosis and counseling of patients with hearing loss is necessary to reduce the rate of hearing aid return. In addition, patients must be prescribed the best hearing aid for their condition and advised on how to optimize its operation. In addition to ongoing consultation, regular follow up after hearing rehabilitation may help to reduce the rate of return.

\section{Acknowledgments}

This work was supported by the National Research Foundation of Korea (NRF) grant funded by the Korea government (MSIP) (No. 2011-0030072).

\section{Conflicts of interest}

The authors have no financial conflicts of interest.

\section{REFERENCES}

1) Dalton DS, Cruickshanks KJ, Klein BE, Klein R, Wiley TL, Nondahl DM. The impact of hearing loss on quality of life in older adults. Gerontologist 2003;43:661-8.

2) Healthy Hearing. Mild Hearing Loss: BTE Hearing Aids Are A Perfect Fit [cited 2012 July 12]. Available from: http://www.healthyhearing.com/content/articles/Hearing-aids/Types-of-hearing-aids/ 47505.

3) Khairi Md Daud M, Noor RM, Rahman NA, Sidek DS, Mohamad A. The effect of mild hearing loss on academic performance in primary school children. Int J Pediatr Otorhinolaryngol 2010;74:67-70.

4) Stelmachowicz PG, Pittman AL, Hoover BM, Lewis DE, Moeller MP. The importance of high-frequency audibility in the speech and language development of children with hearing loss. Arch Otolaryngol Head Neck Surg 2004;130:556-62.

5) Choi J, Chung WH. Age-related hearing loss and the effects of hearing aids. J Korean Med Assoc 2011;54:918-24.

6) National Evidence-based healthcare Collaborating Agency. Analysis of hearing aid effectiveness and obstacles of the domestic hearing loss patients. Seoul: National Evidence-based healthcare Collaborating Agency;2011. p.1-10.

7) Purdy S, Williams W. Guideline for diagnosing occupational noiseinduced hearing loss. Part 3: Audiometric standards. Purdy \& Williams: Guidelines for audiometry for diagnosis of NIHL [cited 2016
May 7]. Available from: http://www.acc.co.nz/PRD EXT CSMP/ groups/external_communications/documents/reference_tools/wpc 091007.pdf

8) Wuyts FL, Van de Heyning PH, Declau F. Audiometric criteria for linkage analysis in genetic hearing. Chapter 9. In: Stephens D, Read A, Martini A, editors. Developments in Genetic Hearing Impairment. London: Whurr Publishers;1998. p.54-9.

9) Demeester K, van Wieringen A, Hendrickx JJ, Topsakal V, Fransen E, van Laer L, et al. Audiometric shape and presbycusis. Int J Audiol 2009;48:222-32.

10) Korea Research Institute for Local Administration. A study on share and management system of welfare finance for aged society. Seoul: Korea Research Institute for Local Administration;2006. Available from: http://www.krila.re.kr/common/filedown.asp?ix $=7221$.

11) Kochkin S, Beck DL, Christensen ALA, Medwetsky L, Northern J, Sweetow R. MarkeTrak VIII: Why consumers return hearing aids: A guide for reducing hearing aid returns. Washington, DC: Better Hearing Institute;2012.

12) Chang YS, Choi J, Park GY, Youm HY, Byun HY, Cho YS. Evaluation of satisfaction with hearing aids using a questionnaire based on markeTrak survey. Korean J Otorhinolaryngol-Head Neck Surg 2014; 57:304-13.

13) Hearing Loss - Mild. Only Slightly Imperfect Ears by Jamie Berke [cited 2016 Jun 28]. Available from: https://www.verywell.com/hearing-loss-mild-1046139.

14) Cruickshanks KJ, Wiley TL, Tweed TS, Klein BE, Klein R, MaresPerlman JA, et al. Prevalence of hearing loss in older adults in Beaver Dam, Wisconsin. The Epidemiology of Hearing Loss Study. Am J Epidemiol 1998;148:879-86.

15) Ries PW. Prevalence and characteristics of persons with hearing trouble: United States, 1990-91 (Vital and Health Statistics, Series 10, No. 188; DHHS Publication No. PHS 94-1516). Hyattsville, MD: National Center for Health Statistics;1994.

16) Cajade Frías J, Labella Caballero T. [Epidemiological aspects of otosclerosis (1). Its frequency in comparison with other ear pathologies, incidence and prevalence]. An Otorrinolaringol Ibero Am 2003;30:115.

17) Alexander TH, Harris JP. Current epidemiology of Meniere's syndrome. Otolaryngol Clin North Am 2010;43:965-70.

18) Jerger J, Chmiel R, Stach B, Spretnjak M. Gender affects audiometric shape in presbyacusis. J Am Acad Audiol 1993;4:42-9.

19) Sanchez L. The epidemiology of tinnitus. Audiol Med 2004;2:8-17.

20) Axelsson A, Ringdahl A. Tinnitus--a study of its prevalence and characteristics. Br J Audiol 1989;23:53-62.

21) Spoendlin H. Retrograde degeneration of the cochlear nerve. Acta Otolaryngol 1975;79:266-75.

22) Disabled World. Which Type of Hearing Aid is Best [cited 2013 Jun 16]. Available from:http://www.disabled-world.com/disability/types/ hearing/communication/types-hearing-aids.php.

23) Mayo Clinic. Hearing aids: How to choose the right one [cited 2014 Nov 7]. Available from: http://www.mayoclinic.org/diseases-conditions/hearing-loss/in-depth/hearing-aids/art-20044116. 